\section{Dr. Sybill Storz mit dem Bundesverdienstkreuz ausgezeichnet}

_ Im November erhielt die Geschäftsführerin der Karl Storz GmbH, Dr. Sybill Storz, das Bundesverdienstkreuz am Bande. Der Ministerpräsident des Landes Baden-Württemberg, Stefan Mappus, der Sybill Storz die Auszeichnung überreichte, würdigte im Rahmen des Festaktes insbesondere ihre Verdienste um die Wirtschaft und das Gemeinwohl. Denn Sybill Storz, die seit dem Tod ihres Vaters 1996 das Unternehmen Karl Storz erfolgreich, mit Weitsicht und einem Sinn sowohl für Nachhaltigkeit als auch für Innovationen führt, zeichnet sich nicht nur durch unternehmerische Leistungen aus, sie setzte sich in den vergangenen
Jahren und Jahrzehnten tatkräftig für soziale und kulturelle Projekte ein. Zudem unterstützt sie weltweit die medizinische Fortbildung, um vor allem weniger privilegierten Menschen Zugang zu effektiver medizinischer Versorgung zu ermöglichen. „Mit ihrem ausgeprägten sozialen und gesellschaftlichen Verantwortungsbewusstsein haben Sie sich herausragende Verdienste um das Wohlergehen des Landes und der Gesellschaft erworben“, würdigte Ministerpräsident Mappus das vielschichtige Engagement der Preisträgerin.

Nach Informationen der Karl Storz GmbH

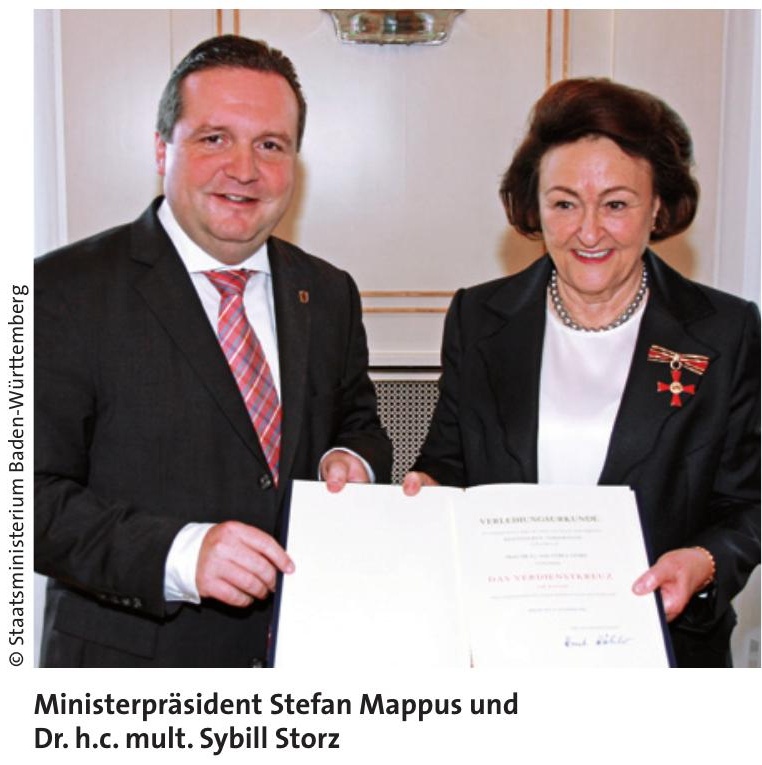

\title{
Mometason: wirksam, sicher und kostengünstig
}

- Allergische Rhinitis, akute Rhinosinusitis und Nasenpolypen gehören zu den häufigsten HNO-Erkrankungen. Gemeinsam ist den drei Erkrankungen die Entzündungsreaktion, sagte Prof. Claus Bachert, Gent/ Belgien.

Unabhängig von der Krankheitsgenese führt eine antientzündliche Behandlung mit intranasalen Kortikosteroiden (INS) zu einem deutlichen Rückgang der Symptome. Die Präparate sind dabei anderen Medikamenten meist überlegen. In den ARIA-Leitlinien von 2008 [Bousquet et al.] werden topische Kortikoide deshalb als Mittel der ersten Wahl in der Behandlung der allergischen Rhinitis empfohlen. Auch bei der akuten und chronischen Rhinosinusitis mit
Nasenpolypen können nasale Kortikoide auf höchstem Evidenzniveau eingesetzt werden - so die EP3OS-Leitlinien von 2007 [Fokkens et al.].

Ein besonders gutes Nutzen-Risiko-Profil unter den nasalen Kortikoiden hat Mometason-Nasenspray (Nasonex ${ }^{\circledR}$ ), sagte Bachert. Das Präparat ist für die Behandlung von Erwachsenen und Kindern ab sechs Jahren zugelassen und hat bei sehr starker Entzündungshemmung eine besonders geringe Bioverfügbarkeit.

Nasonex ${ }^{\circledR}$ hat außerdem im Vergleich zu einem vermeintlich preisgünstigeren $\mathrm{Bu}$ desonid-haltigen Nasenspray eine höhere Kosten-Effektivität, so Prof. Ludger Klimek, Wiesbaden. Dies zeigt sich anhand einer kürzlich publizierten PharmakoökonomieStudie bei niedergelassenen Ärzten. Anhand der Verordnungsdaten vom Oktober 2004 bis September 2008 ergaben sich für Mometason Therapiekosten von 24,70 Euro pro Jahr. im Vergleich dazu lagen die Kosten für das Budesonid-Spray bei 34,04 Euro. Der Grund dafür dürfte vor allem in den geringeren Verbrauchsmengen des modernen Kortikoid-Sprays sowie den geringeren Folgeverordnungen liegen. bk

Pressekonferenz „Effektivität im Fokus und Kosten im Blick: Aktuelle Aspekte der nasalen Steroidtherapie mit Nasonex ${ }^{\circledR}$ anlässlich des 5. Deutschen Allergiekongresses, Hannover, 9. September 2010. Veranstalter: Essex Pharma

\section{Das „Hörwunder von Mannheim“ fand offene Ohren}

— Die Charity-Aktion „Hörwunder von Mannheim“, die der Hörimplantate-Hersteller Cochlear im Rahmen der 44. Fortbildungsveranstaltung für HNO-Ärzte durchführte, erwies sich als voller Erfolg. „Schenken Sie uns Ihr Ohr; gemeinsam machen wir etwas Wunderbares daraus!" war das Motto der Aktion. Dazu waren alle Kongressteilnehmer eingeladen, am Messestand der Firma ihre Ohren fotografieren zu lassen, und sehr viele folgten der Einla- dung. Aus den hunderten Ohren-Fotos entstand in der Zeit des Kongresses eine große, vom Computer animierte Collage im Pop-Art-Stil.

Das originelle Projekt diente dem guten Zweck, die Anschaffung einer SoundfieldAnlage in der Mannheimer HermannGutzmann-Schule für hörgeschädigte und sprachbehinderte Kinder zu finanzieren. Für jedes fotografierte Ohr spendet der Initiator Cochlear nämlich drei Euro. Die dringend benötigte Soundfield-Anlage verstärkt während des Unterrichts die Stimme des Lehrers und erleichtert den Kindern so das Lernen. „Zahlreiche Besucher haben unsere Foto-Aktion tatkräftig unterstützt, so dass 1.700 Euro Spendengeld für die Hermann-GutzmannSchule zusammenkamen", freute sich Thomas Topp, Leiter der Cochlear Deutschland $\mathrm{GmbH} \&$ Co KG.

gz

Nach Informationen der Cochlear $\mathrm{GmbH}$ 